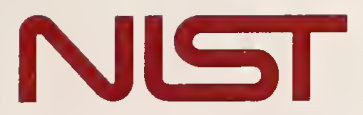

National Institute of Standards and Technology

Technology Administration, U.S. Department of Commerce

NAT'L INST, OF STAND \& TECH

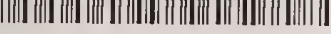

Alllob 46025B

NIST

PUBLICATIONS

NIST Technical Note 1539

\title{
Angular Errors in Polarimetric Radar Cross Section Calibration Using a Rotating Dihedral
}

\author{
Lorant A. Muth \\ Catherine Johnson \\ Donald Morales \\ Timothy Conn
}

$Q C$
100
45753
41539
2005
$c .2$




\section{Angular Errors in Polarimetric Radar Cross Section Calibration Using a Rotating Dihedral}

Lorant A. Muth

Electromagnetics Division

Electronics and Electrical Engineering Laboratory

National Institute of Standards and Technology

325 Broadway

Boulder, Colorado 80305-3328

Catherine Johnson

Donald Morales

Timothy Conn

EG\&G Technical Services, Inc.

National Radar Cross Section Test Facility

46TG/TGR

Holloman Air Force Base, NM 88330

April 2005

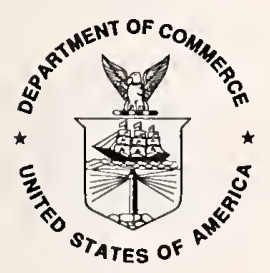

U.S. DEPARTMENT OF COMMERCE, Carlos M. Gutierrez, Secretary 
Certain commercial entities, equipment, or materials may be identified in this document in order to describe an experimental procedure or concept adequately. Such identification is not intended to imply recommendation or endorsement by the National Institute of Standards and Technology, nor is it intended to imply that the entities, materials, or equipment are necessarily the best available for the purpose.

National Institute of Standards and Technology Technical Note 1539

Natl. Inst. Stand. Technol. Tech. Note 1539, 20 pages (April 2005)

CODEN: NTNOEF

For sale by the Superintendent of Documents, U.S. Government Printing Office Internet: bookstore.gpo.gov Phone: (202) 512-1800 Fax: (202) 512-2250 


\title{
ANGULAR ERRORS IN POLARIMETRIC RADAR CROSS SECTION CALIBRATION USING A ROTATING DIHEDRAL
}

\author{
Lorant A. Muth, Catherine Johnson, ${ }^{*}$ \\ Donald Morales, ${ }^{*}$ and Timothy Conn* \\ Electronics and Electrical Engineering Laboratory \\ National Institute of Standards and Technology \\ 325 Broadway \\ Boulder, Colorado 80305-3328
}

\begin{abstract}
We examine how accurately a radar cross section measurement system's transmit and receive parameters can be determined by use of a rotating dihedral as the polarimetric calibration device. We derive expressions for the errors due to misalignment in the angle of rotation of the dihedral. In addition, we obtain expressions for the angles $\alpha_{0, h v}$ and $\alpha_{0, v h}$ for which the measured cross-polarization ratios of a target vanish. Since the theoretical cross-polarization ratio of a cylinder is 0 , we can thus find the calibration bias-correction angles. We use both simulated and real data to demonstrate the robustness of this bias-angle correction technique. We also derive expressions for the uncertainty in the polarimetric system parameters.
\end{abstract}

Keywords: angular errors, calibration, polarimetric radar cross section, rotating dihedrals

\section{THEORETICAL FORMULATION}

In general, the receive matrix in a polarimetric RCS measurement system is given by

$$
\left(\begin{array}{ll}
r_{h h} & r_{h v} \\
r_{v h} & r_{v v}
\end{array}\right)
$$

where $r_{p q}$ is the $p$-polarized electric field received when a $q$-polarized electric field is illuminating the target. Here $p$ and $q$ indicate the horizontal $h$ or the vertical $v$ polarizations. Equation (1) can be normalized so that the receive matrix becomes

$$
r=\left(\begin{array}{cc}
1 & \epsilon_{h} \\
\epsilon_{v} & 1
\end{array}\right)=\tilde{t}
$$

\footnotetext{
* EG\&G Technical Services, Inc., at National Radar Cross Section Test Facility, 46TG/TGR, Holloman Air Force Base, NM 88330
} 
where $t$ is the transmit matrix for reciprocal systems, and the cross-polarization ratios are defined as

$$
\epsilon_{h}=\frac{r_{h v}}{r_{h h}},
$$

and

$$
\epsilon_{v}=\frac{r_{v h}}{r_{v v}}
$$

We represent the error-free receive matrix as

$$
r_{0}=\left(\begin{array}{cc}
1 & \epsilon_{h, 0} \\
\epsilon_{v, 0} & 1
\end{array}\right)=\tilde{t}_{0}
$$

which has the inverse

$$
r_{0}^{-1}=\left(\begin{array}{cc}
1 & -\epsilon_{h, 0} \\
-\epsilon_{v, 0} & 1
\end{array}\right) /\left|r_{0}\right|=\tilde{t}_{0}^{-1}
$$

where $\left|r_{0}\right|$ is the determinant,

$$
\left|r_{0}\right|=1-\epsilon_{h, 0} \epsilon_{v, 0}
$$

We calibrate the polarimetric measurement system with a rotating dihedral [1], which has the high-frequency scattering matrix

$$
D(\theta)=k_{D}\left(\begin{array}{cc}
-\cos 2 \theta & \sin 2 \theta \\
\sin 2 \theta & \cos 2 \theta
\end{array}\right)
$$

where $\theta$ (with respect to the vertical) is the angle of rotation of the dihedral about the line-of-sight from the radar to the dihedral; $k_{D}$ depends on the size of the dihedral and can be obtained computationally. The measured signal is

$$
M^{(d)}=k k_{D} r D(\theta) t
$$

where $k$, a phase-dependent constant, can be determined by a measurement on a cylinder whose radar cross cross section has been computed accurately. The superscript ${ }^{(d)}$ in eq (4) and elsewhere means that the quantity is derived from measured data. We write the matrix components of eq (4) explicitly as

$$
M^{(d)}=k k_{D}\left(\begin{array}{cc}
\left(-1+\epsilon_{h}^{2}\right) \cos 2 \theta+2 \epsilon_{h} \sin 2 \theta & \left(\epsilon_{h}-\epsilon_{v}\right) \cos 2 \theta+\left(1+\epsilon_{h} \epsilon_{v}\right) \sin 2 \theta \\
\left(\epsilon_{h}-\epsilon_{v}\right) \cos 2 \theta+\left(1+\epsilon_{h} \epsilon_{v}\right) \sin 2 \theta & \left(1-\epsilon_{v}^{2}\right) \cos 2 \theta+2 \epsilon_{v} \sin 2 \theta
\end{array}\right) .
$$

Let

$$
\theta=\theta_{0}+\alpha,
$$

where $\alpha$ is the error in the angle of rotation. For $\alpha=0$, the ratios $r_{h, 0}^{(d)}$ and $r_{v, 0}^{(d)}$ of the Fourier coefficients of the matrix components $M_{h h}^{(d)}$ and $M_{v v}^{(d)}$ are

$$
r_{h, 0}^{(d)}=\frac{1}{2} \frac{\epsilon_{h, 0}^{2}-1}{\epsilon_{h, 0}}
$$


and

$$
r_{v, 0}^{(d)}=\frac{1}{2} \frac{1-\epsilon_{v, 0}^{2}}{\epsilon_{v, 0}}
$$

For $\alpha \neq 0$, these ratios are

$$
r_{h, \alpha}^{(d)}=\frac{\left(\epsilon_{h, 0}^{2}-1\right) \cos 2 \alpha+2 \epsilon_{h, 0} \sin 2 \alpha}{2 \epsilon_{h, 0} \cos 2 \alpha+\left(1-\epsilon_{h, 0}^{2}\right) \sin 2 \alpha}
$$

and

$$
r_{v, \alpha}^{(d)}=\frac{\left(1-\epsilon_{v, 0}^{2}\right) \cos 2 \alpha+2 \epsilon_{v, 0} \sin 2 \alpha}{2 \epsilon_{v, 0} \cos 2 \alpha+\left(\epsilon_{v, 0}^{2}-1\right) \sin 2 \alpha} .
$$

For known $r_{h, 0}^{(d)}$ and $r_{v, 0}^{(d)}$ (obtained from the Fourier coefficients of the data), we can readily show by substitution that

$$
\epsilon_{h, 0}, \text { and }-\frac{1}{\epsilon_{h, 0}}
$$

and

$$
\epsilon_{v, 0}, \quad \text { and }-\frac{1}{\epsilon_{v, 0}}
$$

are solutions to eqs (6-7). We note that these solution pairs are negative reciprocals of each other. In practice, we want a radar system with cross-polarization ratios less than unity. Without loss of generality, we assume that $\epsilon_{h, 0}<<1$ and $\epsilon_{v, 0}<<1$. The solutions to eqs (8-9) in terms of the solutions of eqs (6-7) are

$$
\epsilon_{h, \alpha}=\left\{\begin{array}{c}
\frac{\left(1+\epsilon_{h, 0}^{2}\right) \sin 2 \alpha+2 \epsilon_{h, 0}}{\left(1+\epsilon_{h, 0}^{2}\right) \cos 2 \alpha+1-\epsilon_{h, 0}^{2}}, \\
\frac{\left(1+\epsilon_{h, 0}^{2}\right) \sin 2 \alpha-2 \epsilon_{h, 0}}{\left(1+\epsilon_{h, 0}^{2}\right) \cos 2 \alpha-1+\epsilon_{h, 0}^{2}},
\end{array}\right.
$$

and

$$
\epsilon_{v, \alpha}=\left\{\begin{array}{l}
\frac{-\left(1+\epsilon_{v, 0}^{2}\right) \sin 2 \alpha+2 \epsilon_{v, 0}}{\left(1+\epsilon_{v, 0}^{2}\right) \cos 2 \alpha+1-\epsilon_{v, 0}^{2}}, \\
\frac{-\left(1+\epsilon_{v, 0}^{2}\right) \sin 2 \alpha-2 \epsilon_{v, 0}}{\left(1+\epsilon_{v, 0}^{2}\right) \cos 2 \alpha-1+\epsilon_{v, 0}^{2}} .
\end{array}\right.
$$

We can readily verify that the solution pairs in eqs (12-13) are negative reciprocals of each other, as in the error-free case. For $\alpha=0$, the first solutions in eqs (12-13) reduce to $\epsilon_{h, 0}$ and $\epsilon_{v, 0}$, respectively; and for $\alpha \approx 0$, these same solutions are still less than unity.

If a target's scattering matrix is given by

$$
T=\left(\begin{array}{cc}
t_{h h} & t_{h v} \\
t_{v h} & t_{v v}
\end{array}\right)
$$


then the error-free received signal from the target is

$$
M_{t}^{(d)}=k^{\prime} r_{0} T \tilde{r}_{0}
$$

where $k^{\prime}$ is a complex constant that depends on the distance $r$ between the target and the radar. If the target is at the same location as the dihedral, then $k^{\prime}=k$, as in eq (4). However, we do not know $r_{0}$, the error-free receive matrix, since there is always an angular bias error in a calibration with a rotating dihedral. Equation (15) can be inverted to obtain an error-contaminated target scattering matrix $T_{\alpha}$, when we use the known error-contaminated system receive and transmit matrices

$$
r_{\alpha}=\left(\begin{array}{cc}
1 & \epsilon_{h, \alpha}^{(1)} \\
\epsilon_{v, \alpha}^{(1)} & 1
\end{array}\right)=\tilde{t}_{\alpha}
$$

Here the superscript ${ }^{(1)}$ indicates that we are using the first solutions in eqs (12-13). These solutions let us recover the error-free target matrix in eq (15), if we set $\alpha=0$.

The target's error-contaminated scattering matrix obtained from the measurements $M_{t}^{(d)}$ is then

$$
k^{\prime} T_{\alpha}=r_{\alpha}^{-1} M_{t}^{(d)} \tilde{r}_{\alpha}^{-1} .
$$

We can expand the components of $T_{\alpha}$ in a second-order Taylor series to exhibit explicitly the dependence on the small error-parameter $\alpha$. The details of these expansions are given in the appendix.

Here, we concentrate on the cross-polarization ratios,

$$
\tau_{h h, \alpha}^{h v} \equiv \frac{t_{h v, \alpha}}{t_{h h, \alpha}}
$$

and

$$
\tau_{v v, \alpha}^{v h} \equiv \frac{t_{v h, \alpha}}{t_{v v, \alpha}}
$$

which can be used to determine the small angular-bias error $\alpha$ present in the calibration procedure that has been propagated into the system cross-polarization ratios $\epsilon_{h, \alpha}$ and $\epsilon_{v, \alpha}$. Again, to simplify these ratios for small errors $\alpha$, we expand them in a second-order Taylor series of the form

$$
\frac{t_{h v, \alpha}}{t_{h h, \alpha}}=\frac{t_{h v, 0}}{t_{h h, 0}}+\alpha Q_{h v, 0}+O\left(\alpha^{2}\right),
$$

and similarly for $\tau_{v v, \alpha}^{v h}$. The explicit forms of $Q_{h v, 0}$ and $Q_{v h, 0}$ in terms of all the error-free system and target parameters are to be found in the appendix.

An important special case of eq (20) is when the cross-polarization ratio on the left vanishes. We then solve for $\alpha_{0, h v}$ :

$$
\alpha_{0, h v}=\frac{1-\epsilon_{h, 0} \epsilon_{v, 0}}{\left(1+\epsilon_{h, 0}^{2}\right) t_{v v, 0}-t_{h h, 0}\left(1+\epsilon_{v, 0}^{2}\right)} t_{h v, 0}+O\left(t_{h v, 0}^{2}\right)
$$


and, similarly,

$$
\alpha_{0, v h}=\frac{1-\epsilon_{h, 0} \epsilon_{v, 0}}{\left(1+\epsilon_{h, 0}^{2}\right) t_{v v, 0}-t_{h h, 0}\left(1+\epsilon_{v, 0}^{2}\right)} t_{v h, 0}+O\left(t_{v h, 0}^{2}\right) .
$$

Theoretically, both eqs (21) and (22) should give the same angle $\alpha$ for reciprocal targets with $t_{h v, 0}=t_{v h, 0}$. However, in practice we might obtain slightly different results due to system noise and target misalignments. We can neglect all second-order terms in eqs (21-22) to drive a simple zero-order expression for $\alpha$ :

$$
\alpha_{0, h v} \approx \frac{t_{h v, 0}}{t_{v v, 0}-t_{h h, 0}}
$$

which is independent of the system parameters. Similarly, we can easily obtain an expression for $\alpha_{0, v h}$. We have assumed in eq (23) that $t_{v v, 0}-t_{h h, 0} \neq 0$. Note that for a sphere this condition is not satisfied; however, a sphere is not depolarizing, therefore, $t_{h v, 0} \approx 0$, and, hence, $\alpha$ is undefined.

\section{SIMULATIONS}

We used computer simulations to verify the analysis presented in the previous section and to demonstrate that the bias angle $\alpha$, which represents the angular misalignment between the calibration dihedral and a target, can be accurately determined from measurements.

In particular, we designed the simulations to understand eq (23) in two ways:

(1) explore the range of $\alpha$ for which eq (23) recovers the correct value, and

(2) explore the sensitivity of eq (23) to the condition that its denominator must not vanish.

We assume various values for the receive parameters in eq (1), and use eqs (3) and (4) to simulate measured dihedral data. Without any loss of generality, we assume that the product $k k_{d}$ in eq (4) is 1 . We then introduce a range of bias angles $\alpha$ into eq (5) to obtain biased dihedral measurements, and use Fourier analysis to solve for biased system parameters. Next we simulate various target measurements using the scattering matrix defined in eq (14). The biased scattering-matrix elements are recovered using eq (17), and then use eq (23) to recover the known bias angle $\alpha$.

We performed simulations with various cross-polarization ratios $\epsilon_{h}$ and $\epsilon_{v}$ in eq (1c), and various test target matrices $T$ in eq (14). We used the following combinations of parameters:

(1) for the receive and transmit matrices,

$$
r_{1}=\left(\begin{array}{cc}
1 & 0.1 \\
-0.15 & 1
\end{array}\right)=\tilde{t}_{1}
$$

and

$$
r_{2}=\left(\begin{array}{cc}
1 & 0.01 \\
-0.015 & 1
\end{array}\right)=\tilde{t}_{2}
$$

which represent average and good channel isolations, respectively, and 
(2) for the test targets matrices,

$$
\begin{aligned}
T_{1} & =\left(\begin{array}{cc}
0.5 & 0 \\
0 & -1
\end{array}\right), \\
T_{2} & =\left(\begin{array}{cc}
1 & 0 \\
0 & -1
\end{array}\right), \\
T_{3} & =\left(\begin{array}{cc}
0.5 & 0 \\
0 & 1
\end{array}\right), \\
T_{4} & =\left(\begin{array}{cc}
0.8 & 0 \\
0 & 1
\end{array}\right),
\end{aligned}
$$

and

$$
T_{5}=\left(\begin{array}{cc}
0.98 & 0 \\
0 & 1
\end{array}\right)
$$

Since all the off-diagonal scattering matrix elements are 0 , these matrices represent nondepolarizing targets with various copolar ratios that satisfy the condition $t_{v v, 0}-t_{h h, 0} \neq 0$ to different degrees. When the copolar components are nearly equal, as in case $T 5$, we have a sphere-like target and expect the observed (biased) cross-polarization to be very low.

Figures 1 and 2 show the results of simulations. In Figure 1 we see that errors in the computed bias angle are very small in all cases except $T 5$ (the sphere-like target). For a well isolated system $R 2$, these errors are significantly smaller than in the poorly isolated system $R 1$. Overall, however, the errors in the predicted bias angles are insignificant, especially at very small bias angles. For $T 5$, however, the errors quickly become very large. For R1 the error curves are linear in $\alpha$; for the case R2, the presence of second and higher-order terms is apparent.

In Figure 2 we show the measured cross-polarization ratio in decibels as a function of known bias angles $\alpha$ for the cases $T 1 \cdots T 5$. As $\alpha \rightarrow 0$, this measured ratio approaches $-\infty$, which is the correct theoretical value (assumed). The dependence on $\alpha$ is very strong in all cases. The dependence on the ratio of copolar matrix components is also very strong; we get the lowest measured cross-polarization ratios for $T 5$, as expected. The dependence on system isolation ( $R 1$ vs. $R 2$ ) is very weak.

These figures show that our technique to remove angular error biases in polarimetric calibration using a rotating dihedral is robust. The simulations demonstrate that

(1) a wide range of bias angles are recovered accurately, and

(2) even if the the denominator in eq (23) nearly vanishes, we still get satisfactory results. This implies that the correction technique can be applied to measurements on many types of targets.

\section{EXPERIMENTAL DETERMINATION OF ALIGNMENT BIAS}

We have also demonstrated the robustness of our technique using real data. Figure 3 shows the calibration fixture used to rotate the calibration dihedral. The dihedral was aligned with the vertical by means of a manual inclinometer. The rotation was continuous, and the 


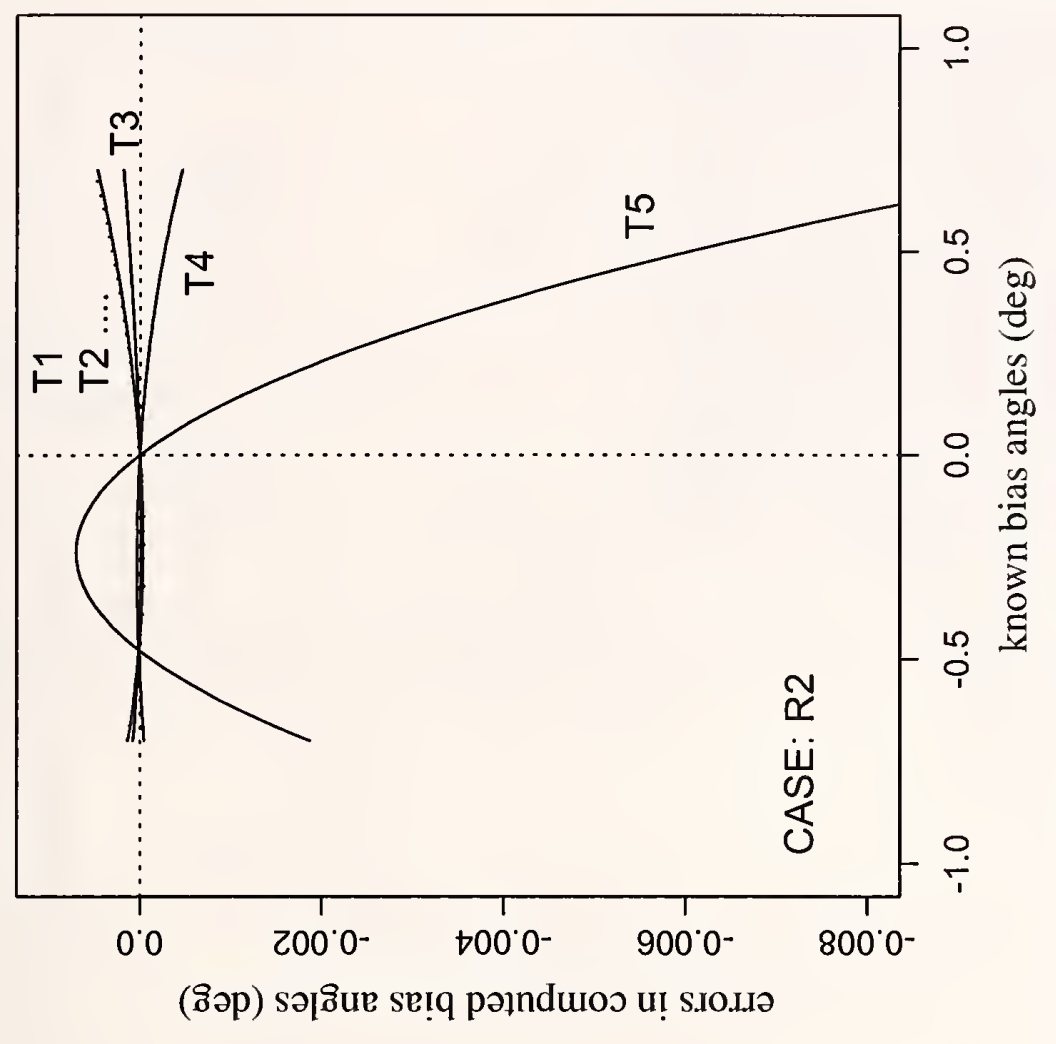

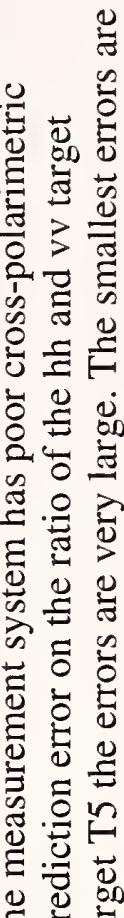
흔 บิ กิ

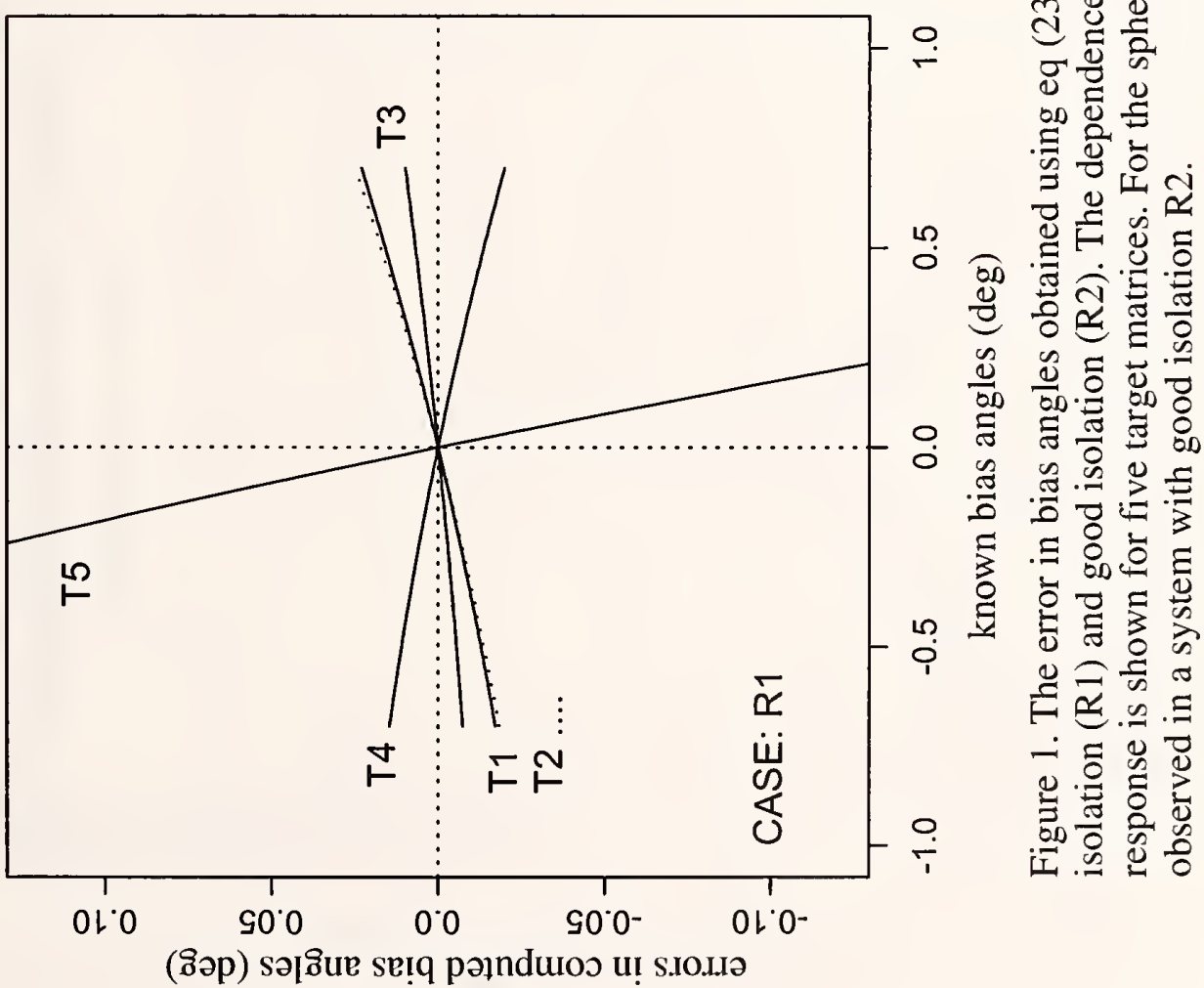




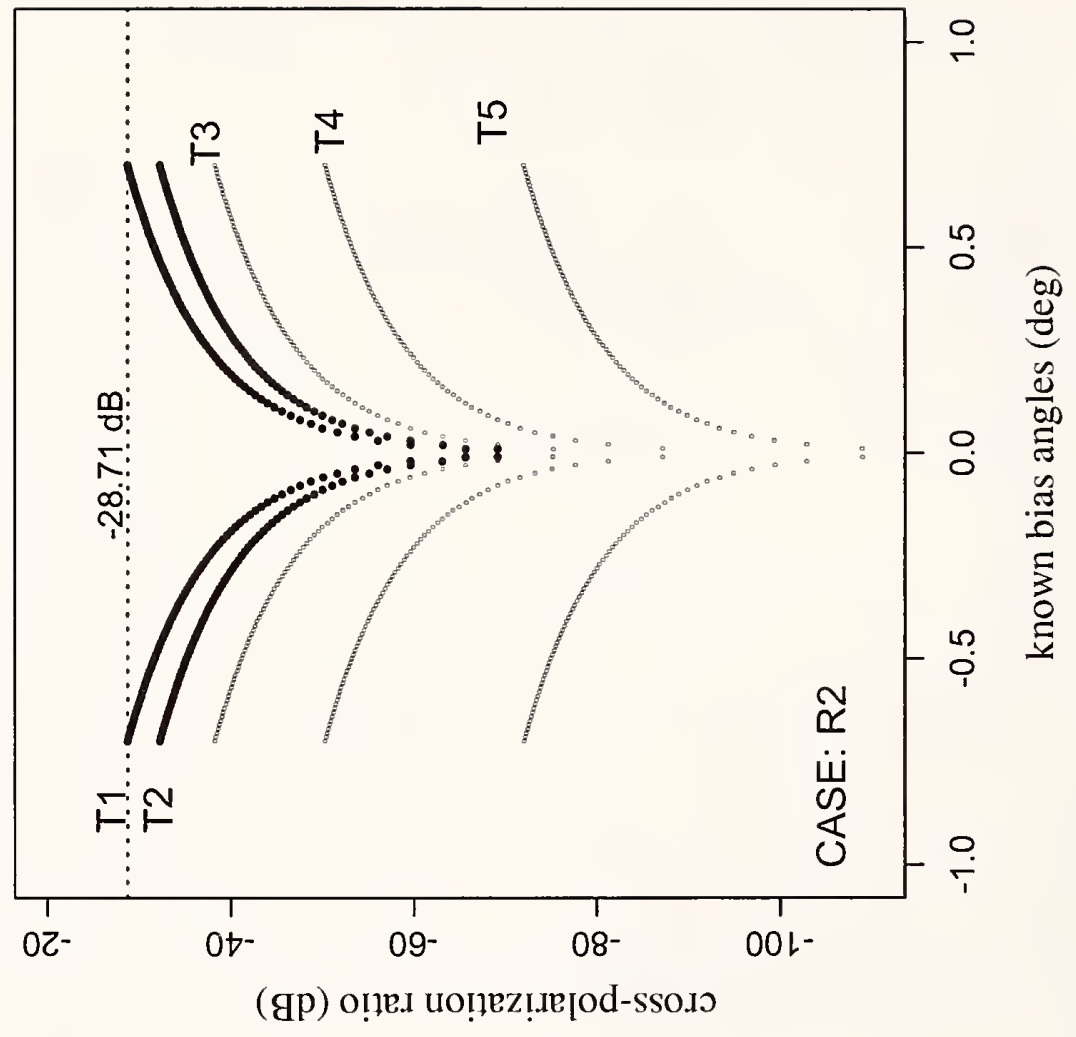

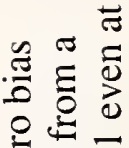

沗的

(क) «

छ

$\dot{0}$

\& $00 \%$

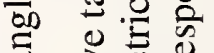

$\geq$ 巳

ฮ ๘

₹ 0 응

오

สี

.

¿

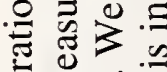

䒕

ᄂ

.유 ప ప

导导莺

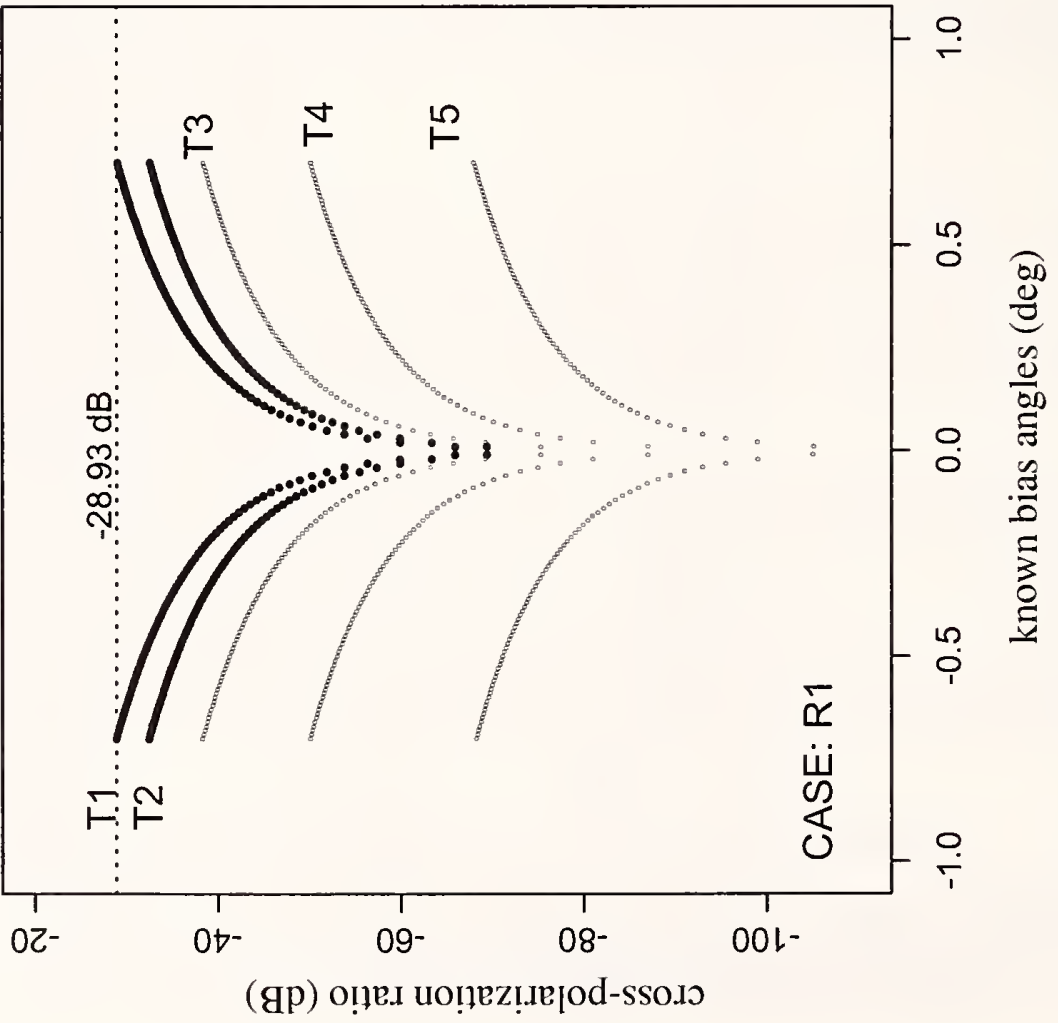

त $\dot{0}$

\&

$\circ$ के

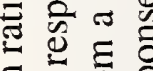

ㅇ. 을 응

ํㅝㄴ

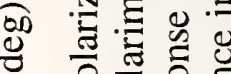

ㅎํㅇ

它造造

की 它

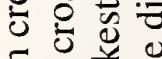

로 휼

803

خ

ฮั

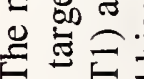

을

고

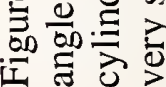

(qp) o!̣e. uo!̣ez!ıejod-sso.ı 


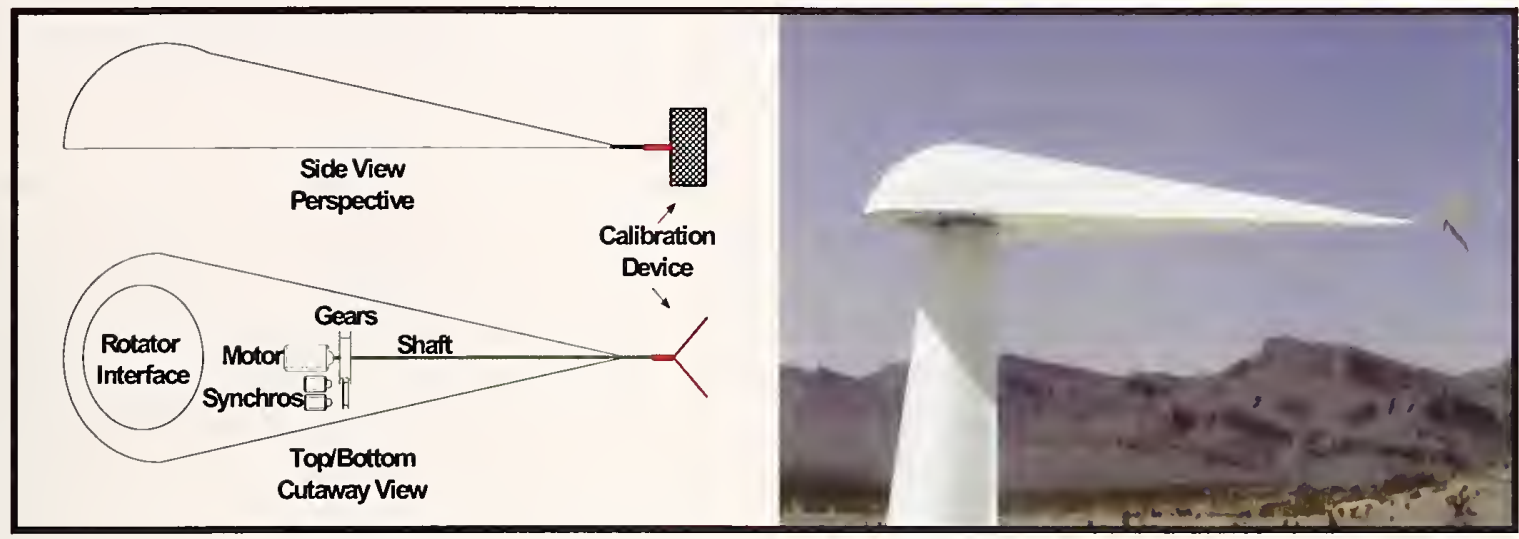

Figure 3. The fixture used to rotate a calibration dihedral for polarimetric measurements to obtain the full transmit-receive matrix of the radar system. The cross-polarization response of the fixture without the dihedral was also measured.

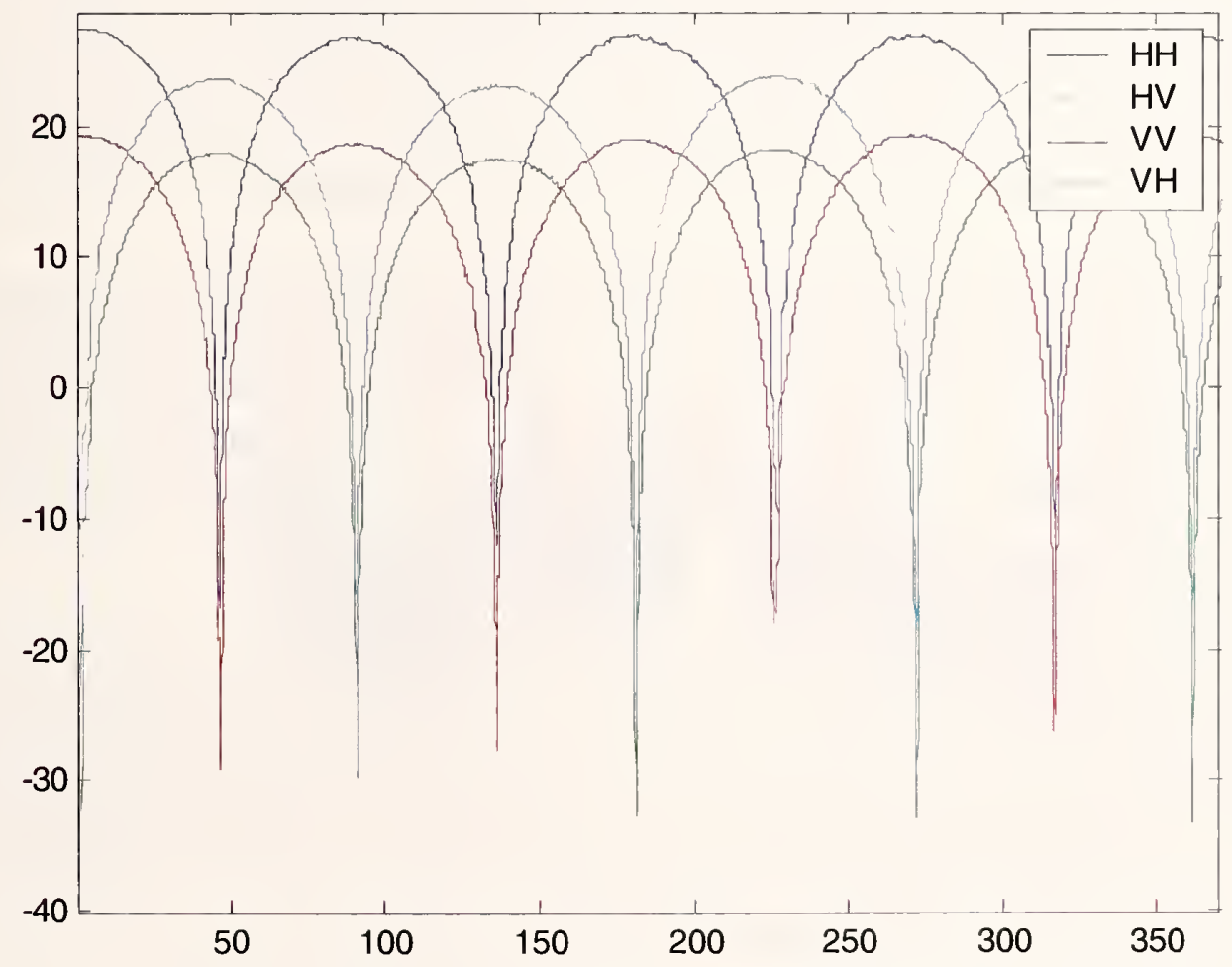

Figure 4. The uncalibrated polarimetric signals at $9.6 \mathrm{GHz}$ received from the rotating dihedral shown in Figure 3. 
angle of rotation was recorded in real time as the radar was transmitting and receiving the calibration pulses. The uncalibrated data, shown in Figure 4, were then used to determine the system transmit and receive parameters using the method developed in Reference [2], which, in principle, is equivalent to using eqs (6), (7) and (17).

Next, measurements were made on a cylinder, because, theoretically, it has zero crosspolarization response. The cylinder was also aligned with the vertical by means of the manual inclinometer. In case of perfect alignment with the dihedral, the measured crosspolarized response of the cylinder would be zero theoretically, or at the level of noise experimentally. We make the realistic assumption that the cylinder is much better aligned than the dihedral. Then, any nonvanishing cross-polar measurement on the cylinder can be attributed to the misalignment of the dihedral. This bias in the rotational angle of the dihedral, relative to the coordinate system in which the cylinder is aligned, is $\alpha$. Any small cross-polarized measurement (beyond noise) on the cylinder indicates the presence of bias, and a succesful correction technique should eliminate this.

The bias-correction angle was determined numerically from the biased calibration data. We start by assuming a value for $\alpha$ and determine the system parameters. Then we compute the cylinder scattering matrix elements. Figure 5 shows the cross-polar $(h v)$ element of the cylinder's scattering matrix as a function of the bias angle. The bias correction angle was determined to be $0.18^{\circ}$, compared to $0.19^{\circ}$ calculated from eq (23). The $0.01^{\circ}$ difference is consistent with the error curves presented in Figure 1. When we compare the crosspolar ratio levels at $\alpha=0.2$ in Figure 2 and at the bias-corrected angle $\alpha^{\prime}=0$ in Figure 5 , we can estimate that we are dealing with a measurement system configured similarly to case $R 2 ; T 2$. However, case $\mathrm{R} 1$ cannot be eliminated conclusively with such a simple comparison.

In Figure 6 we show the co-polar and cross-polar $v h$ scattering matrix elements of the supporting test target (shown in Figure 3) as a function of azimuthal rotation. The two lower curves show the computed cross-polar responses before and after the calibration bias angle has been removed from the data to obtain bias-free system parameters. We observe a significantly lower cross-polar ratio, in agreement with computational predictions.

\section{CALIBRATION UNCERTAINTY}

After the calibration data have been corrected for the bias, expressions (A8) through (A12) can be used to estimate the uncertainties in the scattering matrix elements of the target, and expressions (A13) and (A14) can be used to estimate the uncertainties in the system parameters. We let the $\alpha=0$ terms be the bias-corrected values, and redefine $\alpha$ as the estimated upper bound of uncertainy in the computed bias angle obtained using eq (23).

The main sources of uncertainty in $\alpha$ are:

(1) computational errors, especially due to the approximations used,

(2) the presence of noise in the measurements, and

(3) drift.

Special techniques can be used to correct the data for drift by exploiting the rotational symmetries of the dihedral [3, Section 4]. We will not discuss the details here, but assume 


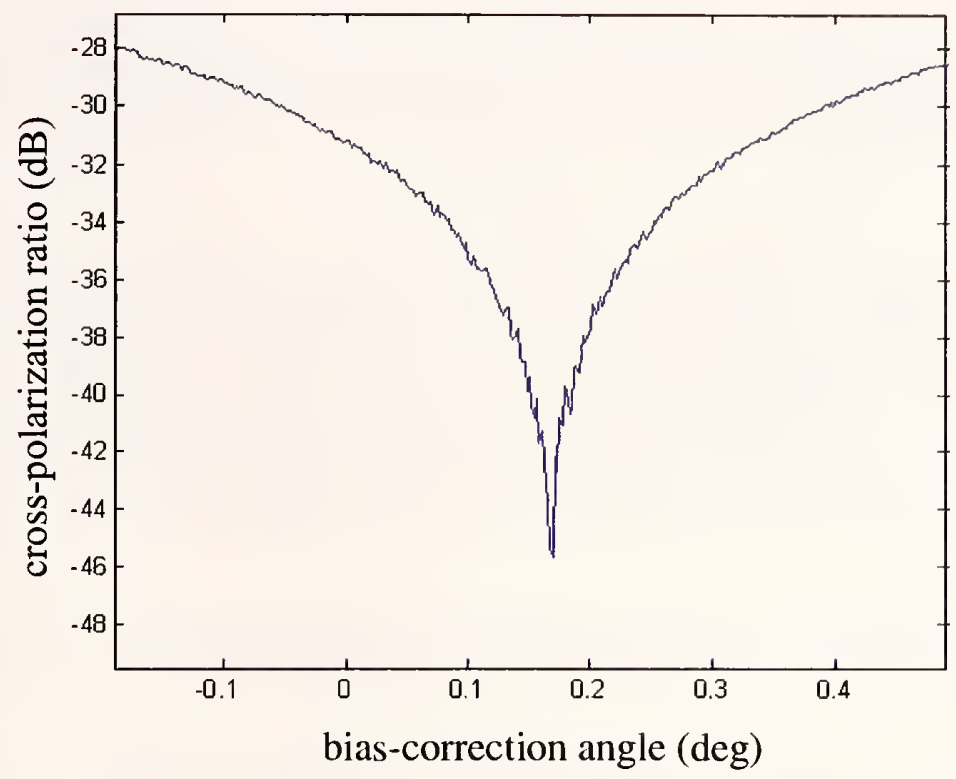

Figure 5. The measured cross-polarization ratio (decibels) of a cylinder as a function of the bias-correction angle. The deep null indicates that the calibration bias angle has been removed to obtain bias-free system parameters.

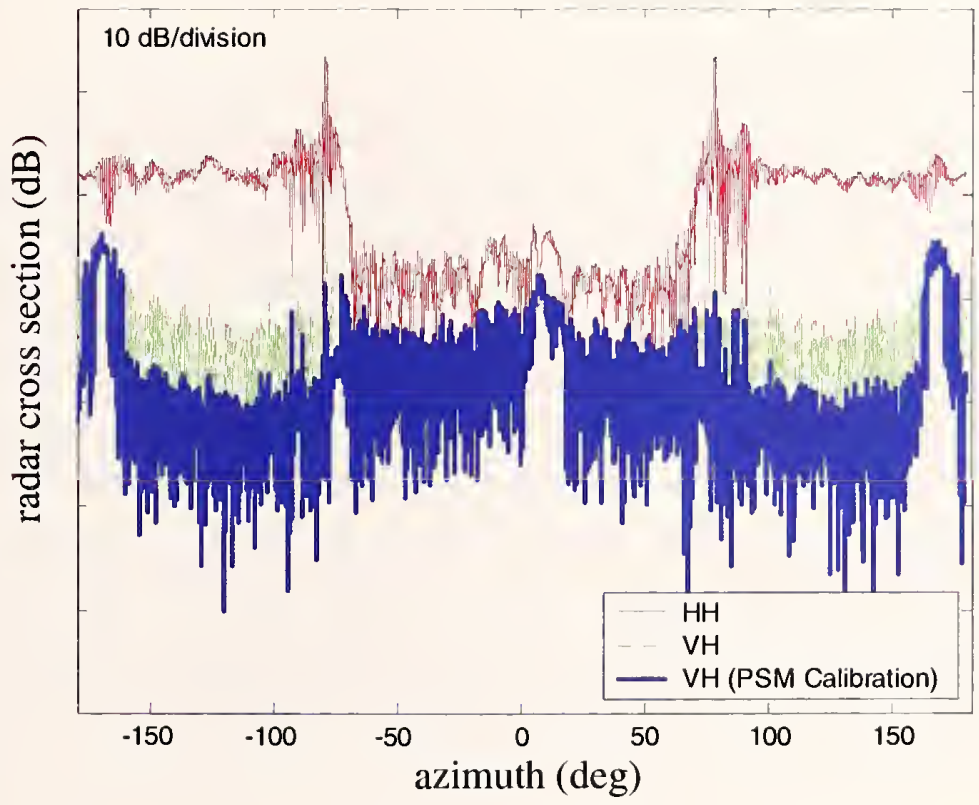

Figure 6. The polarimetric response of the test body shown in Figure 3 as a function of azimuthal rotation. The top curve is the HH co-polar response; the middle and bottom curves are the cross-polar responses without and with calibration bias-angle correction, respectively. 
that the effect of drift has been adequately removed. According to Figure 1, we can estimate the upper bound of computational errors $\delta \alpha \approx \alpha / 10$, which is then used as the estimated uncertainty in $\alpha$. To account for the effect of noise, we include a coverage factor of 2 and round upwards. Hence, in our simulations,

$$
\delta \alpha \equiv \pm \alpha / 5
$$

For example, in Figure 5 the bias-correction angle was found to be $\alpha \approx 0.18^{\circ}$; hence, $\delta \alpha=0.04^{\circ}$, and $\alpha=0.18^{\circ} \pm 04^{\circ}$. This estimate is valid for systems with parameters in the range of $\mathrm{R} 1$ to $\mathrm{R} 2$ presented in Figure 1. To determine the uncertainties in the measurements, we use the first-order expressions (A8) through (A13) with $\alpha= \pm \delta \alpha$, and substitute the bias-corrected measurements for all quantities with 0 subscripts. For the case $R 2: T 2$, we get

$$
t_{h h}=1 \pm 0.000007 ; t_{h v}=0 \pm 0.0007 ; t_{v h}=0 \pm 0.0007 ; t_{v v}=-1 \pm 0.00001
$$

and

$$
\epsilon_{h}=0.01 \pm 0.0004 ; \epsilon_{v}=-0.015 \pm 0.0004
$$

We emphasize that the simple method of estimating the uncertainty $\delta \alpha$ in $\alpha$ presented here need not be adopted. Any fully described method, with well-defined operational steps, supported with clearly stated and easily defensible assumptions, would be adequate to the task.

\section{SUMMARY AND CONCLUSION}

We have presented a theoretical procedure to remove angular misalignment errors in the calibration of polarimetric RCS measurement systems. The calibration is accomplished with a rotating dihedral which might not be perfectly aligned in the vertical direction. We define the vertical as the vertical alignment of a calibration cylinder. In practice, the cylinder can be aligned with a manual inclinometer much more accurately than can a dihedral. We exploit the vanishing of the cross-polar response of a cylinder to derive a simple expression for the misalignment bias angle. The results of theoretical simulations agree with real measurements. We show excellent agreement between the bias angles obtained numerically and theoretically using the expression derived in this study. The expressions derived for the target polarimetric scattering matrix components and for the system parameters as functions of the bias angle can also be used to obtain the corresponding uncertainties by use of an estimated upper-bound uncertainty in the bias angle. The results indicate that the theoretical bias-correction technique presented in this study is very robust.

This work was supported by the DoD Triservice Calibration Coordination Group (CCG). Special thanks are extended to H. Glick, M. Cunevalis, and R. Cleland of the Microwave Subgroup for their long-term support of this project. 


\section{REFERENCES}

[1] L. A. Muth, R. C. Wittman and R. L. Lewis, "Polarimetric calibration of reciprocal antenna radars," Antenna Measurement Techniques Assoc. (AMTA) 1995 Proceedings, 1995.

[2] M. W. Whitt and F. T. Ulaby, "A polarimetric radar calibration technique with insensitivity to target orientation," Radio Science, vol. 25, pp. 1137 - 1143, 1990.

[3] L. A. Muth, P. D. Domich, B. M. Welsh, A. L. Buterbaugh and B. M. Kent, "Polarimetric calibration standards for monostatic radar systems," Proceedings of the 1998 Workshop and Symposium, NCSL, 1998. 


\section{APPENDIX}

Here we explicitly exhibit the matrix elements of $T_{\alpha}$ in terms of the error-free system parameters to a first order in $\alpha$ :

$$
\begin{gathered}
t_{h h, \alpha}=t_{h h, 0}-\alpha \frac{2 \epsilon_{h, 0}\left(1+\epsilon_{v, 0}^{2}\right) t_{h h, 0}+\left(t_{h v, 0}+t_{v h, 0}\right)\left(1+\epsilon_{h, 0}^{2}\right)}{1-\epsilon_{v, 0} \epsilon_{h, 0}}+O\left(\alpha^{2}\right), \\
t_{h v, \alpha}=t_{h v, 0}+ \\
\alpha \frac{\left(\left(1+\epsilon_{h, 0}^{2}\right) \epsilon_{v, 0}-\epsilon_{h, 0}\left(1+\epsilon_{v, 0}^{2}\right)\right) t_{h v, 0}+\left(1+\epsilon_{v, 0}^{2}\right) t_{h h, 0}-t_{v v, 0}\left(1+\epsilon_{h, 0}^{2}\right)}{1-\epsilon_{v, 0} \epsilon_{h, 0}}+O\left(\alpha^{2}\right), \\
t_{v h, \alpha}=t_{v h, 0}+ \\
\alpha \frac{\left(\left(1+\epsilon_{h, 0}^{2}\right) \epsilon_{v, 0}-\epsilon_{h, 0}\left(1+\epsilon_{v, 0}^{2}\right)\right) t_{v h, 0}+\left(1+\epsilon_{v, 0}^{2}\right) t_{h h, 0}-t_{v v, 0}\left(1+\epsilon_{h, 0}^{2}\right)}{1-\epsilon_{v, 0} \epsilon_{h, 0}}+O\left(\alpha^{2}\right),
\end{gathered}
$$

and

$$
t_{v v, \alpha}=t_{v v, 0}+\alpha \frac{2 \epsilon_{v, 0}\left(1+\epsilon_{h, 0}^{2}\right) t_{v v, 0}+\left(t_{v h, 0}+t_{h v, 0}\right)\left(1+\epsilon_{v, 0}^{2}\right)}{1-\epsilon_{v, 0} \epsilon_{h, 0}}+O\left(\alpha^{2}\right) .
$$

The $h v$ cross-polarization ratio is, to a first order in $\alpha$ (see eq (20)),

$$
\frac{t_{h v, \alpha}}{t_{h h, \alpha}}=\frac{t_{h v, 0}}{t_{h h, 0}}+\alpha Q_{h v, 0}+O\left(\alpha^{2}\right)
$$

and a similar expression can be written for the $v h$ polarization.

Explicitly, $Q_{h v, 0}$ is given by

$$
\begin{aligned}
& \left(1-\epsilon_{h, 0} \epsilon_{v, 0}\right) Q_{h v, 0}=1+\epsilon_{v, 0}^{2}-\left(1+\epsilon_{h, 0}^{2}\right) \frac{t_{v v, 0}}{t_{h h, 0}} \\
& \quad+\left[\epsilon_{h, 0}\left(1+\epsilon_{v, 0}^{2}\right)+\epsilon_{v, 0}\left(1+\epsilon_{h, 0}^{2}\right)\right] \frac{t_{h v, 0}}{t_{h h, 0}}+\left(1+\epsilon_{h, 0}^{2}\right)\left(\frac{t_{h v, 0}}{t_{h h, 0}} \frac{t_{v h, 0}}{t_{h h, 0}}+\frac{t_{h v, 0}^{2}}{t_{h h, 0}^{2}}\right)
\end{aligned}
$$

and $Q_{v h, 0}$ is given by

$$
\begin{aligned}
& \left(1-\epsilon_{h, 0} \epsilon_{v, 0}\right) Q_{v h, 0}=-\left(1+\epsilon_{h, 0}^{2}\right)+\left(1+\epsilon_{v, 0}^{2}\right) \frac{t_{h h, 0}}{t_{v v, 0}} \\
& \quad-\left[\epsilon_{h, 0}\left(1+\epsilon_{v, 0}^{2}\right)+\epsilon_{v, 0}\left(1+\epsilon_{h, 0}^{2}\right)\right] \frac{t_{v h, 0}}{t_{v v, 0}}-\left(1+\epsilon_{v, 0}^{2}\right)\left(\frac{t_{v h, 0}}{t_{v v, 0}} \frac{t_{h v, 0}}{t_{v v, 0}}+\frac{t_{v h, 0}^{2}}{t_{v v, 0}^{2}}\right) .
\end{aligned}
$$

We observe that the leading terms in eqs (A5) and (A6) are

$$
1-\frac{t_{v v, 0}}{t_{h h, 0}}, \text { and }-1+\frac{t_{h h, 0}}{t_{v v, 0}}
$$


which is consistent with eq (23). For a dihedral, the ratios in eq (A7) evaluate to -1 .

For a system with good polarimetric isolation, second-order terms can be safely neglected. The target scattering matrix elements then simplify as

$$
\begin{gathered}
t_{h h, \alpha} \approx t_{h h, 0}\left(1-2 \alpha \epsilon_{h, 0}\right)-\alpha\left(t_{h v, 0}+t_{v h, 0}\right)+O\left(\alpha^{2} ; \epsilon^{2}\right), \\
t_{h v, \alpha} \approx t_{h v, 0}\left(1+\alpha\left(\epsilon_{v, 0}-\epsilon_{h, 0}\right)\right)+\alpha\left(t_{h h, 0}-t_{v v, 0}\right)+O\left(\alpha^{2} ; \epsilon^{2}\right), \\
t_{v h, \alpha} \approx t_{v h, 0}\left(1+\alpha\left(\epsilon_{v, 0}-\epsilon_{h, 0}\right)\right)+\alpha\left(t_{h h, 0}-t_{v v, 0}\right)+O\left(\alpha^{2} ; \epsilon^{2}\right),
\end{gathered}
$$

and

$$
t_{v v, \alpha} \approx t_{v v, 0}\left(1+2 \alpha \epsilon_{v, 0}\right)+\alpha\left(t_{v h, 0}+t_{h v, 0}\right)+O\left(\alpha^{2} ; \epsilon^{2}\right) .
$$

The cross-polarization ratios simplify similarly; for example,

$$
\frac{t_{h v, \alpha}}{t_{h h, \alpha}} \approx \frac{t_{h v, 0}}{t_{h h, 0}}+\alpha\left(1-\frac{t_{v v, 0}}{t_{h h, 0}}+\left(\epsilon_{h, 0}+\epsilon_{v, 0}\right) \frac{t_{h v, 0}}{t_{h h, 0}}\right)+O\left(\alpha^{2} ; \epsilon^{2} ; t^{2}\right) .
$$

If in eq (A12) we further neglect terms with $\epsilon$-s and set the left side to 0 , we obtain eq (23).

Finally, we obtain the sytem parameters to first order in $\alpha$ from eqs (12-13):

$$
\epsilon_{h, \alpha}=\epsilon_{h, 0}+\alpha\left(1+\epsilon_{h, 0}^{2}\right)+O\left(\alpha^{2}\right),
$$

and

$$
\epsilon_{v, \alpha}=\epsilon_{v, 0}-\alpha\left(1+\epsilon_{v, 0}^{2}\right)+O\left(\alpha^{2}\right) .
$$





\section{NIST Technical Publications}

\section{Periodical}

Journal of Research of the National Institute of Standards and Technology-Reports NIST research and development in metrology and related fields of physical science, engineering, applied mathematics, statistics, biotechnology, and information technology. Papers cover a broad range of subjects, with major emphasis on measurement methodology and the basic technology underlying standardization. Also included from time to time are survey articles on topics closely related to the Institute's technical and scientific programs. Issued six times a year.

\section{Nonperiodicals}

Monographs-Major contributions to the technical literature on various subjects related to the Institute's scientific and technical activities.

Handbooks-Recommended codes of engineering and industrial practice (including safety codes) developed in cooperation with interested industries, professional organizations, and regulatory bodies.

Special Publications-Include proceedings of conferences sponsored by NIST, NIST annual reports, and other special publications appropriate to this grouping such as wall charts, pocket cards, and bibliographies.

National Standard Reference Data Series-Provides quantitative data on the physical and chemical properties of materials, compiled from the world's literature and critically evaluated. Developed under a worldwide program coordinated by NIST under the authority of the National Standard Data Act (Public Law 90-396). NOTE: The Journal of Physical and Chemical Reference Data (JPCRD) is published bimonthly for NIST by the American Institute of Physics (AIP). Subscription orders and renewals are available from AIP, P.O. Box 503284, St. Louis, MO 63150-3284.

Building Science Series-Disseminates technical information developed at the Institute on building materials, components, systems, and whole structures. The series presents research results, test methods, and performance criteria related to the structural and environmental functions and the durability and safety characteristics of building elements and systems.

Technical Notes-Studies or reports which are complete in themselves but restrictive in their treatment of a subject. Analogous to monographs but not so comprehensive in scope or definitive in treatment of the subject area. Often serve as a vehicle for final reports of work performed at NIST under the sponsorship of other government agencies. Voluntary Product Standards-Developed under procedures published by the Department of Commerce in Part 10 , Title 15, of the Code of Federal Regulations. The standards establish nationally recognized requirements for products, and provide all concerned interests with a basis for common understanding of the characteristics of the products. NIST administers this program in support of the efforts of private-sector standardizing organizations. Order the following NIST publications-FIPS and NISTIRs-from the National Technical Information Service, Springfield, VA 22161.

Federal Information Processing Standards Publications (FIPS PUB)-Publications in this series collectively constitute the Federal Information Processing Standards Register. The Register serves as the official source of information in the Federal Government regarding standards issued by NIST pursuant to the Federal Property and Administrative Services Act of 1949 as amended, Public Law 89-306 (79 Stat. 1127), and as implemented by Executive Order 11717 (38 FR 12315, dated May 11,1973) and Part 6 of Title 15 CFR (Code of Federal Regulations).

NIST Interagency or Internal Reports (NISTIR)-The series includes interim or final reports on work performed by NIST for outside sponsors (both government and nongovernment). In general, initial distribution is handled by the sponsor; public distribution is handled by sales through the National Technical Information Service, Springfield, VA 22161, in hard copy, electronic media, or microfiche form. NISTIR's may also report results of NIST projects of transitory or limited interest, including those that will be published subsequently in more comprehensive form. 
U.S. Department of Commerce

National Institute of Standards and Technology

325 Broadway

Boulder, Colorado 80305-3328

\section{Official Business}

Penalty for Private Use, $\$ 300$ 